\title{
Sonntagsdienst am 11. März 2002
}

\section{J. Küttel}

\section{Allgemeines}

Ich bin Pädiater in der Luzerner Vorortsgemeinde Ebikon. Die Gegend, wo sich Luzern gegen Zug hin öffnet, heisst Rontal, benannt nach dem kleinen Flüsschen, das aus dem Rotsee fliesst. Wir sind zwölf Dienstärzte - ich der einzige Pädiater - und versorgen etwa 25000 Einwohner. Ein Arzt hat jeweils 24 Stunden Notfalldienst. Der Dienst wechselt täglich und läuft übers ganze Jahr. Das gibt total 30-32 Dienste, davon 10-12 Sonn-, Feieroder Samstage. Bis 60 macht jeder 100\% Dienst, dann nur noch freiwillig. An einem Sonntag oder Feiertag und ab Samstagmittag ist der Dienstarzt der einzige sicher erreichbare Arzt. Jeder «kann» «alles». Strassenunfälle werden in der Regel direkt durch die Ambulanz des nahen Kantonsspitals versorgt. Es gibt keine zentrale Anlaufstelle. Das Kantonsspital verweist auf den Notfallarzt und führt kein direkt zugängliches Ambulatorium. Lediglich das Kinderspital nimmt Selbstzuweisungen öfters an. Es existiert ein gut organisierter und für $\mathrm{Pa}-$ tienten direkt zugänglicher augenärztlicher Notfalldienst $4 \mathrm{~km}$ entfernt in der Stadt Luzern und ein psychiatrischer Notfalldienst, der aber nur via Notfallarzt und nicht direkt für Patienten zugänglich ist. Ertragsmässig sieht es so aus: eine reine Konsultation in der Praxis von 15 Minuten Dauer ohne Labor und Medikamente kostet am Sonntag Fr. 36.25 und nachts 65.25 .

\section{Chronologie des Tages}

\subsection{0}

Ein strahlender Sonntagmorgen grüsst mich, Frühling in Reinkultur. Ich treffe in der Praxis ein, wo auch meine MPA ist. Für die erste Stunde haben wir vier Patienten eingeschrieben, die nicht anders kommen wollten. So der 10 Jahre alte Etienne, der wegen seines ADS während der Woche die Sonderschule besucht, Melanie, die die Desensibilisierung vorgestern verpasste, und Vanessa, welche die frisch operierten eingewachsenen Zehennägel zeigt.

\subsection{0}

Julia hat Nasenbluten, die Eltern rufen an. Wir sagen ihnen, was zu tun ist und falls es so nicht stoppt, soll sie kommen. Es stoppt. Der Vater holt noch Nasensalbe.

\subsection{5}

Die 14jährige Bilijana ging vorgestern ohne Sonnenschutz auf den Skitag. Nun hat sie ein verschwollenes Gesicht und Blasen. Sie bekommt eine Kortisonlotion und die eindringliche Empfehlung, solche Strahlenunfälle der Haut künftig zu vermeiden.

\subsection{0}

Eine Mutter und ihr vierjähriger Sohn kommen wegen Gliederschmerzen und Fieber. Die Mutter hat gerade eine Tripel-Therapie gegen Helicobacter angefangen und will wissen, ob Paracetamol dazu vertragen werde. Ja. Wie lange denn die Grippe gehe, sie sei so elend dran. Spätestens in einer Woche ist es vorbei. Vielleicht sei sie nachher noch eine gewisse Zeit etwas müde.

\subsection{5}

Die 6 Jahre alte Rasma hat eine Otitis und eine juckende Epizoonose, Antibiotikadiskussion und Calpred auf die Haut. 3 Tage später läuft das Ohr aus, nun gibt's halt doch ein Antibiotikum. Die neulich empfohlene Strategie bei Otitis media erleidet wieder mal Schiffbruch.

\subsection{0}

Ein 52 Jahre alter Käser mit Psoriasis hat - «weil es mich so juckt» - seine Krusten am Knie aufgekratzt und nun einen Weichteilinfekt mit Bursitis. Er fiebert. Noch Grippe dazu? Die Lymphknoten in der Leiste sind in Ordnung. Er bekommt Amoxizillin. Morgen zum Hausarzt, für Labor vielleicht und Kontrolle.

\subsection{5}

Eine 50jährige Frau kommt mit den letzten Austrittsberichten unter dem Arm - diverse kardiologische Operationen wegen eines Aortenwurzelaneurismas. Seit 4 Tagen hat sie Schulterschmerzen links und nahm ein NSAR. Ihr Kardiologe ist nicht erreichbar. Ich untersuche sie und finde nichts Gravierendes; der Schmerz kommt wohl eher vom Skelett her. Sicher weiss ich es nicht. Ich schicke sie zwecks Beurteilung ins Spital. Nur kein Risiko eingehen!

\subsection{0}

Eine 45 Jahre alte Frau - die Mutter eines 22jährigen Sohns mit Neugeborenen-Herpes-Enzephallitis - zeigt sich wegen Nackenschmerzen, Fieber 
und Husten seit Tagen. Sie dachte an Meningitis, ich an Grippe mit Pneumonie. Auskultation und Thorax sind normal, also doch eher viral.

\subsection{5}

Eine 22 Jahre alte Flight-Attendant war dieses Jahr schon 3mal eine Woche in Mombasa. Weil die Edelweiss nur ein Flugzeug habe, werde jeweils die Crew ausgetauscht und gleich zurückgeflogen. Im eisgekühlten Flugzeug habe sie sich wohl ihre Schulterschmerzen und das starke Kopfweh geholt. Nein, eine Malaria-Prophylaxe mache man wegen einer Woche nicht. Doch, sie werde von den Biestern immer gestochen. Nein, Fieber habe sie keines, aber dieses Kopfweh sei trotz Ibuprofen kaum zum Aushalten. Muskelhartspann der Schultergegend, Leber nicht vergrössert, nur etwas empfindlich. Lunge normal. CRP und Blutbild normal, keine Thrombopenie. Wahrscheinlich doch nur Zugluft. Soll doch morgen noch zum Tropenarzt, wegen der Malaria. Sie geht und es ist nichts.

\subsection{0}

ist es inzwischen. Ich habe 15 Patienten gesehen an diesem Morgen. Nun rufe ich meine Frau an, schalte das Alibiphon ein und fahre heim. Vielleicht habe ich Glück und 2 Stunden Pause liegen drin. Meine MPA geht spazieren.

\subsection{0}

Zwei kleine Patienten gesehen, nichts Spezielles. Dann noch zwei weitere Kinder mit einer Bronchitis, die sich am Morgen schon angemeldet hatten. Anschliessend noch ein 12 Monate alter Bub mit einer leichten Otitis. Er bekommt Ponstan und zeigt sich in zwei Tagen wieder.

\subsection{0}

Ein 10 Jahre alter Bub hatte vor zwei Tagen einen Bienenstich in den Daumen. Nun hat er einen roten Streifen bis zur Axille und die Eltern befürchten eine Blutvergiftung. Die Lymphknoten sind normal und Fieber hat er auch keins. Sicher nur toxisch und kein Infekt. Ich gebe lediglich Claritin, weil es so furchtbar juckt und zeige ihm noch, wie man Bienenstachel entfernt, ohne den Giftsack noch ganz auszupressen. Sicherheitshalber Kontrolle in 3 Tagen oder sofort bei Fieber. Es heilt alles schön ab, der Termin wird gestrichen.

\subsection{5}

Der vier Jahre alte Joris hat Asthma. Es ist nicht das erste Mal. Er bekommt die Inhalationstherapie und den Abklärungstermin für nächste Woche.

\subsection{0}

Ein 18 Monate altes Kind hat seit dem Vortag über $39^{\circ}$ Fieber und die Eltern wollen in 5 Tagen nach Japan. Ich untersuche es ganz und finde keinen Infektherd. Auch CRP, BB und Urin sind normal. Symptomatische Therapie mit Paracetamol und Beruhigung der Eltern.

\subsection{5}

Eine 40jährige Lehrerin hat einen «Nervenzusammenbruch», es sei zuviel passiert in den letzten Wochen. Sie möchte ein Gespräch und Medikamente und es wäre ihr jetzt egal zu sterben. Draussen warten schon drei Leute, ich habe doch keine Zeit und bin auch nicht Psychiater. Zum Glück gibt es den konsiliarischen Notfallpsychiater. Nach ein paar Versuchen klappt es und sie kann nach Luzern zur Kollegin. Bin ich froh! Und sie auch.

\subsection{0}

In der Zwischenzeit habe ich eine weitere Grippepatientin gesehen, eine Pronation douloureuse reponiert - gute Reklame im vollen Wartezimmer, da die Leute das Kind vorher und nachher sahen -, eine Frau mit Urticaria behandelt, eine SA-positive Angina, einen Buben mit Streptokokkenanitis (später mit positivem Abstrich bestätigt) und einen Mann mit Wadenmuskelzerrung während einer Skitour gesehen. Dann noch ein Kind mit einem möglichen Fremdkörper vom Vortag im linken Auge, den erst der Augenarzt am nächsten Tag fand (abends um halb acht rufe ich ihn nicht mehr an, es ging ja letzte Nacht auch).

\subsection{0}

Der momentan letzte Patient ist 14 Monate alt und hat gerade mal seit 2 Stunden Fieber bis $40^{\circ}$. Ich untersuche ihn ganz und finde «nichts». Dann ruft noch einer mit Zahnweh an. Immerhin holt er das Schmerzmittel in der Apotheke in Luzern.

\subsection{5}

Meine MPA eröffnet mir, dass sie das mit dem Notfalldienst nicht mehr lange mitmache, das sei ein furchtbarer Stress, wir müssten das diskutieren. Dem Stress stimme ich bei und diskutieren werden wir es auch. Eine Änderung steht nicht in Aussicht. Es war schon viel schlimmer. Es ging schon mal an einem Sonntag bis 21.30 so weiter. Sie geht nun nach Hause und ich warte auf den nächsten Patienten, der mit dem Velo stürzte. Anfänglich wollte seine Frau einen Hausbesuch; ob ich denn die RQW am Ellbogen nicht daheim nähen könne, die Schulter tue ihm 
furchtbar weh und er könne kaum gehen. Er kam schliesslich in die Praxis. Die Klavikula war frakturiert und der Humeruskopf auch nicht mehr dort, wo er sein sollte. Die RQW war noch das wenigste. Er wurde im Spital noch in der gleichen Nacht operiert.

\subsection{5}

Bis jetzt habe ich 30 Patienten gesehen. Ich faxe noch alle Rückmeldungen an die Kollegen, lüfte die Praxis und bespreche den Anrufbeantworter mit einem «restriktiven» Text. (Das hat mir schon einen Rüffel der Notfalldienstkommission eingetragen.)

\subsection{0}

Todmüde und erschöpft kehre ich heim. Eigentlich wäre es nun genug, aber Ende ist erst in 11 Stunden. Nach dem Nachtessen versuche ich etwas fernzusehen, aber nicke immer wieder ein. Zwischendurch mal etwas, das sich telefonisch erledigen lässt.

\subsection{0}

Angespannt finde ich den Schlaf und höre das Telefon im Traum. Echt läutet es aber erst um 01.15. Der Freund einer jungen Frau ruft an: seit 2 Stunden hat sie Unterleibsschmerzen und
Schmierblutungen. Da bin ich nun wirklich überfordert. Ich rate ihr $\mathrm{zu}$ einem einfachen Schmerzmittel und warmen Wickeln. Sollte es schlimmer werden oder Angst aufkommen, müsse ein Gynäkologe konsultiert werden. Ich sage ihm, wie seine Freundin zu diesem kommt.

\subsection{0}

Der Notfalldienst ist zu Ende. In der Nacht bin ich eigentlich noch gnädig weggekommen. Ich bin wieder in der Praxis. Es ist Montagmorgen und ein normaler Arbeitstag beginnt.

\section{Epilog}

Solche Dienste sind nicht etwa die Ausnahme. Sie leisten einen entscheidenden Beitrag zum Burn-out. Die Situation ist frustrierend, amtlich verordnet und von der Ärztegesellschaft und ihren Organen sanktioniert. Übermüdete und fachlich überforderte Dienstärzte sind auch eine Gefahr für die Patienten. Die am 1. Mai im Bahnhof Luzern eröffnete Permanence könnte uns entlasten und einen Beitrag zur Hebung unserer Lebensqualität bilden. Wünschbar ist auch ein besserer Tarif für Sonn- und Feiertage sowie die Nachtzeit. Mit TARMED wird's besser. Wann? 